Check for updates

Cite this: J. Anal. At. Spectrom., 2021, 36,2649

Received 3rd August 2021

Accepted 24th September 2021

DOI: $10.1039 / \mathrm{d} 1 \mathrm{ja} 00274 \mathrm{k}$

rsc.li/jaas

\section{Laboratory-scale in situ X-ray absorption spectroscopy of a palladium catalyst on a compact inverse-Compton scattering $\mathrm{X}$-ray beamline $\uparrow$}

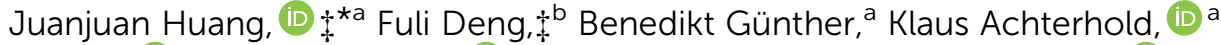 \\ Yue Liu, (iD bc Andreas Jentys, (D)*b Johannes A. Lercher, ${ }^{\mathrm{b}}$ Martin Dierolf (iD ${ }^{\mathrm{a}}$ \\ and Franz Pfeiffer ${ }^{\text {ade }}$
}

X-ray absorption spectroscopy (XAS) is one of the most important techniques used for catalyst characterization, and is often conducted under in situ conditions. Such experiments are typically carried out at synchrotrons, despite the significant improvements in laboratory-based XAS systems in recent years. The limited spectral flux density of X-ray tubes restricts these setups to ex situ or in situ experiments conducted over a large time scale, especially for X-ray energies above $15 \mathrm{keV}$. In this paper, we present an in situ experiment performed on a palladium-based catalyst at the Munich Compact Light Source (MuCLS), a compact synchrotron facility based on inverse Compton X-ray scattering (ICS). Its brilliant, lowdivergence, energy-tuneable X-ray beam makes such a compact facility well suited for XAS techniques, especially with in situ experiments. In this study, we investigated a hydrogen-pretreated $\mathrm{Pd} / \mathrm{C}$ catalyst, which significantly improves the catalytic decarboxylation activity compared to a bare $\mathrm{Pd}(0) / \mathrm{C}$ catalyst. We measured a series of X-ray absorption near-edge structures (XANES) at the Pd K-edge (24.4 keV), with a time resolution of $<5 \mathrm{~min}$ per spectrum. The spectral quality achieved is comparable to spectra obtained from synchrotron measurements. The results infer that the formation of $\alpha$-phase palladium hydride may be the reason for the enhanced performance of the hydrogen-pretreated $\mathrm{Pd} / \mathrm{C}$ catalyst.

\section{Introduction}

Synchrotron-based X-ray spectroscopy techniques enable indepth characterization of materials and chemical systems and are employed in a wide range of technological and research fields. X-ray absorption spectroscopy (XAS) is one of the most commonly used synchrotron techniques, as the measurements supply element-specific information. XAS is divided into two types: X-ray absorption near-edge structure (XANES) and extended X-ray absorption fine structure (EXAFS). ${ }^{\mathbf{1 , 2}}$ Both types

${ }^{a}$ Chair of Biomedical Physics, Department of Physics, School of Natural Sciences and Munich Institute of Biomedical Engineering, Technical University of Munich, 85748 Garching, Germany. E-mail: Juanjuan.Huang@tum.de

${ }^{b}$ Department of Chemistry and Catalysis Research Center, Technical University of Munich, 84747 Garching, Germany.E-mail: Jentys@tum.de

'Shanghai Key Laboratory of Green Chemistry and Chemical Processes, School of Chemistry and Molecular Engineering, East China Normal University, 200062 Shanghai, PR China

${ }^{d}$ Department of Diagnostic and Interventional Radiology, Klinikum Rechts der Isar, Technical University of Munich, 81675 Munich, Germany

${ }^{e}$ Institute for Advanced Study, Technical University of Munich, 85748 Garching, Germany

$\dagger$ Electronic supplementary information (ESI) available. See DOI: 10.1039/d1ja00274k

\$ These authors contributed equally. provide valuable and complementary information on the electronic and geometrical structure of materials.

Since synchrotron radiation first came into use in the 1970 s, ${ }^{3}$ XAS has undergone significant technical and theoretical evolution. Although the first XAS spectra were measured more than 100 years ago on in-house systems, most XAS measurements nowadays are conducted at synchrotrons, due to the high quality and brilliance of the X-rays. However, the limited ability to access such large facilities has inhibited XAS from developing into a routine characterization technique. Therefore, great efforts and improvements have been made in the development of laboratory-based XAS setups.

In contrast to synchrotron facilities, laboratory XAS systems allow for regular on-site characterization of specimens, which is advantageous, especially in an industrial environment. Moreover, a laboratory XAS system removes the need to acquire beamtimes at synchrotrons, which speeds up the pace of academic research. Most of these laboratory setups employ Xray tubes, since they are commercially available, inexpensive, and compact. Modern laboratory setups are either scanningtype systems that use a Rowland focusing geometry, ${ }^{4,5}$ or dispersive-type systems using, e.g., von Hamos geometry. ${ }^{6-9}$ Several commercial prototypes such as the ones developed by easyXAFS, LLC and Sigray, Inc. have even become available in recent years. Details of recent laboratory XAS developments can 
be found in the recent reviews by Zimmermann et al. ${ }^{10}$ and Malzer et al. ${ }^{11}$

A drawback of X-ray sources in XAS is the limited spectral flux density of the bremsstrahlung. This intrinsic limitation restricts such setups to mainly ex situ experiments or in situ experiments over a large time scale. For example, a recent in situ laboratory-based XANES experiment was reported by José G. Moya-Cancino et al., ${ }^{12}$ who studied the slow dynamics (over several tens or hundreds of hours) of a solid $\mathrm{Co} / \mathrm{TiO}_{2}$ FischerTropsch synthesis catalyst.

However, modern XAS is often conducted in an in situ or in operando environment over a much shorter time scale, which is challenging to achieve with low-brilliance X-ray tubes, especially for X-rays above $15 \mathrm{keV}$. Hence, compact X-ray sources based on inverse-Compton scattering (ICS) might be a better alternative to $\mathrm{X}$-ray tubes for in situ XAS in the high-energy range. The performance and cost of such ICS sources are between those of conventional X-ray tubes and synchrotrons. Several projects worldwide thus seek to develop such sources. ${ }^{13-17}$ The Munich Compact Light Source (MuCLS) is one of the already existing ICS facilities and has been in operation since 2015. The X-ray properties of the MuCLS (photon flux of $>10^{10}$ photons per $\mathrm{s}$ in $<5 \%$ bandwidth, energy tunability, low divergence, and relatively small source size) make it well-suited to XAS techniques. We previously reported a first proof-of-principle XAS of the element silver (Ag) at the MuCLS with synchrotron-comparable quality. ${ }^{18}$

In situ XAS is a powerful characterization method used to investigate the mechanism of catalytic processes. Palladium (Pd) is one of the most versatile catalytic metals and has been widely applied in different reactions such as hydrogenation, ${ }^{19-21}$ dehydrogenation $^{22}$ and decarboxylation. ${ }^{23-25}$ In many of these reactions, the formation of palladium hydride $\left(\mathrm{PdH}_{x}\right)$ plays a critical role in catalytic performance. XANES is a very effective method of verifying the existence of $\mathrm{PdH}_{x},{ }^{26-29}$ as the spectral features are directly influenced by the addition of the hydrogen atom.

Here, we conducted an in situ XANES experiment to study the properties of a $\mathrm{Pd} / \mathrm{C}$ catalyst in the decarboxylation reaction of phenylacetic acid to toluene. In this catalytic reaction, it is observed that $\mathrm{H}_{2}$ pretreatment of the $\mathrm{Pd} / \mathrm{C}$ catalyst prior to the reaction can significantly enhance the catalytic performance comparing to bare $\mathrm{Pd} / \mathrm{C}$ catalyst. ${ }^{46}$ The aim of this experiment is to identify the active $\mathrm{Pd}$ species of $\mathrm{Pd} / \mathrm{C}$ while ramping up the temperature towards a reaction level. We measured a series of XANES spectra at Pd K-edge $(24.4 \mathrm{keV})$ at a time resolution of less than 5 min per scan. A dynamic palladium hydride phasetransfer was observed during the temperature ramping process. The excellent spectral quality and good acquisition efficiency unravel the great potential of the setup at the ICS facility for in situ applications, especially at high X-ray energies. Instrumentation, data acquisition, and advantages of XAS at ICS sources are presented in detail.

\section{Experimental}

\section{Experimental setup at the MuCLS}

Fig. 1 shows the MuCLS facility, which is a multipurpose X-ray beamline that utilizes an ICS source. The ICS source was manufactured by Lyncean Technologies Inc., Fremont, USA, while the two endstations (Hutch 1 and Hutch 2) were designed at the Technical University of Munich (TUM). X-rays are generated by the process of inverse Compton scattering (ICS), in which infrared laser pulses collide with MeV electrons head-on. The X-ray energy can be tuned by changing electron energy and is freely adjustable from $15 \mathrm{keV}$ to $35 \mathrm{keV}$. X-ray properties such as flux, source size, and source positions are monitored and stabilized using a specifically designed X-ray beam monitor (XBM) upstream, which cuts off a small portion of the beam for X-ray diagnostics. ${ }^{30}$ More information about the MuCLS facility can be found in the ref. 31 and 32 .

The MuCLS has several specific properties that make it advantageous for XAS techniques: a narrow spectrum with a bandwidth of $3-5 \%$, tunable energy, relatively high flux, and a relatively small source size $(\sim 50 \mu \mathrm{m}, \sigma$-value $)$ and divergence ( $\sim 4 \mathrm{mrad}$, full angle). Routine XAS, unlike other photondemanding spectroscopy techniques such as photon-in/ photon-out spectroscopy, ${ }^{33}$ does not require the full power of a synchrotron. Hence, such a laboratory-scale facility, which fills the gap between conventional X-ray tubes and synchrotrons, is very well suited for XAS techniques. For the Pd K-edge (24.4 keV) XANES measurement, the source was tuned to its $25 \mathrm{keV}$ energy configuration. The total flux of the entire X-ray beam entering Hutch 1 at the time of the experiment was $\sim 1.9 \times 10^{10}$ photons per s with a bandwidth of $\sim 4 \%$.

A specific dispersive setup based on a Laue geometry ${ }^{34,35}$ is adopted for the XAS, illustrated in Fig. 1b. The XAS setup consists of three parts. The first component is a silicon crystal. In this case, a slightly bent $\mathrm{Si}\langle 100\rangle$ crystal wafer ( $200 \mu \mathrm{m}$ thick, $\varnothing$ $=100 \mathrm{~mm}$ ) with a bending radius of $\sim 14 \mathrm{~m}$ was used. The crystal is positioned in a 3D printed home-built crystal holder (see Fig. 1c), which enables the horizontal crystal curvature to be adjusted by applying horizontal pressure to the two edges. The bending radius is estimated from the difference between the theoretical energy range of a flat crystal and the experimental energy range of the bent crystal. ${ }^{18}$ This slight bending curvature is utilized to adjust the X-ray beamsize, energy spread and energy resolution as needed for the experiment.

The XAS setup is currently implemented in Hutch 1, which is located at a distance of $\sim 3 \mathrm{~m}$ to the source and has a total length of $\sim 5 \mathrm{~m}$. The CCD camera is positioned at a distance of $0.45 \mathrm{~m}$ behind the crystal, which leaves plenty of operating space for the sample and its in situ cell in-between. This distance can be increased further depending on the requirements of the experiment. The large experimental hutch and abundant operating space facilitate the implementation of an in situ environment with relatively big sample cells, which may be challenging for some of the X-ray tube-based XAS setups. The crystal is mounted on a stage, which can be aligned in the $x, y, z$-directions and rotated in the $x-y$ plane. The second component is a 2D detector - a XIMEA xiRAY CCD camera (model MH110XCKK-FA, XIMEA GmbH, Münster, Germany) with a $\mathrm{Gd}_{2} \mathrm{O}_{2} \mathrm{~S}: \mathrm{Tb}$ scintillator. It has a pixel size of $9 \mu \mathrm{m}$ and a field-of-view (FOV) of $24 \mathrm{~mm} \times 36 \mathrm{~mm}$ (horizontal $\times$ vertical). The third component is the sample. For the in situ experiments, the sample is 

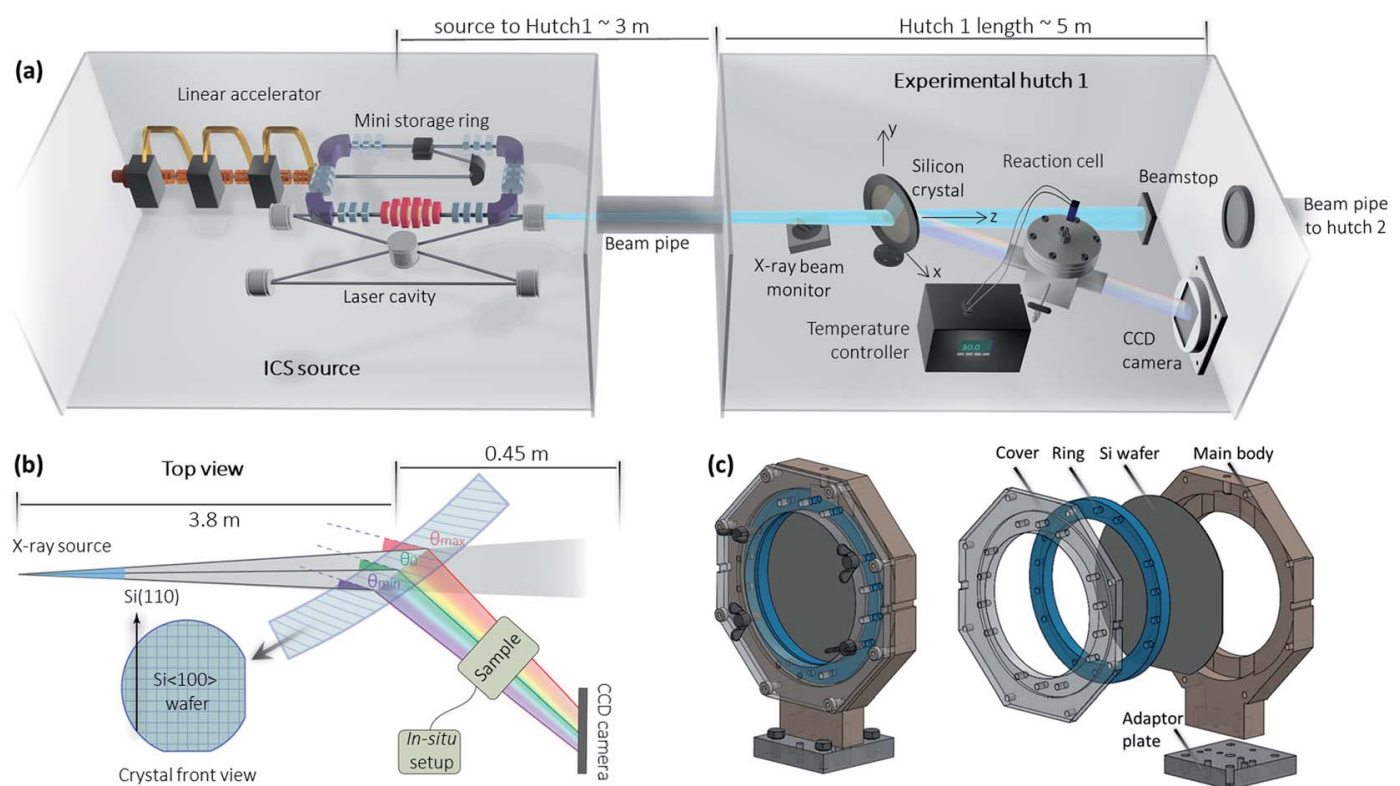

Fig. 1 (a) Schematic of the Munich Compact Light Source (MuCLS) beamline facility and the in situ XAS setup (not to scale). (b) Top-view schematic illustration of the XAS setup, composed of a slightly bent Si $\langle 100\rangle$ wafer, a CCD camera, and the sample contained inside its in situ apparatus. The crystal was rotated so that Si(311) plane was under Bragg condition. The $\sim 4$ mrad X-ray divergence results in a continuous variance of the Bragg angles on the crystal, giving rise to an energy gradient horizontally on the CCD camera. (c) The CAD design of the crystal holder. The crystal can be bent by tightening the screws on the two horizontal edges.

placed in a home-built reaction cell (see Fig. 2), which is connected to a temperature controller.

The crystal is rotated by $\sim 34^{\circ}$ relative to the X-ray beam axis, taking into account the $\mathrm{Si}(311)$ asymmetry angle and the Bragg angle. In dispersive XAS setups at synchrotrons, ${ }^{36}$ samples are generally positioned at the X-ray focus spot that is generated by bending the crystal. In many laboratory XAS setups, ${ }^{6,37}$ samples are placed very close to the X-ray exit window of the tube so as to create a small X-ray spot size on the sample. Our setup uses an unfocused geometry ${ }^{38}$ in which the sample is placed in close proximity to the Laue crystal. As a result, the X-ray beam impinging on the sample is relatively large; for example, the flat field $\left(I_{0}\right)$ shown in Fig. 3 covers a maximum of about 900 pixels $\times 2100$ pixels (horizontal $\times$ vertical), or about $0.8 \mathrm{~cm} \times 1.9 \mathrm{~cm}$ (horizontal $\times$ vertical). Since different energies penetrate through different parts of the sample, an additional correction step is needed to account for sample inhomogeneities. Nevertheless, the setup is straightforward to implement, as the crystal can be easily aligned and removed. On this versatile beamline, this enables rapid switching between different setups for other non-spectroscopy applications such as X-ray imaging techniques. ${ }^{39-42}$ Since no other optics are used, the setup is relatively efficient. Additionally, the absorption of air is relatively low for the X-ray energy employed in the experiments. Consequently, neither vacuum nor helium systems is used in these XAS measurements.

\section{In situ reaction cell}

Fig. 2 shows the schematics of the home-built reaction cell used for the in situ XAS measurement, which was developed at the chemistry department of the Technical University of Munich. It has two Kapton windows, which allow X-rays to pass through. There are two gas tubes with valves, which allow gases to be added for the in situ experiments. The Pd/C powder was compressed into a pellet $(\varnothing=12 \mathrm{~mm})$ and placed inside a small cylindrical holder surrounded by heating wires. The thermocouple and heater are connected to a programmable temperature controller. Thus, the sample cell enables in situ XAS measurement for the $\mathrm{Pd} / \mathrm{C}$ catalyst under certain reaction temperatures and atmospheres.

\section{In situ XAS scans}

The in situ XAS measurement uses a custom XAS scan macro by the experiment control system SPEC (Certified Scientific Software), as illustrated in Fig. 3. Some drifts in the energy position (see Fig. 4a) are observed during XAS experiments at the MuCLS. This might be due to slight movements in the positions of the source or crystal. For this reason, it is necessary to measure a standard Pd foil at the beginning of each scan, not only to calibrate the energy but also to correct any temporal uncertainties. One XAS scan consists of one exposure of the Pd foil, five exposures of the sample $\left(I_{\mathrm{S}}\right)$ to increase the statistics, and one exposure of a flat field $\left(I_{0}\right)$. Each exposure takes $30 \mathrm{~s}$, resulting in a total exposure time of 3.5 min. Including motor movements and other overheads, the total acquisition time of the scan is $4.3 \mathrm{~min}$. In case a better time resolution might be required to observe the dynamic changes during temperature ramping, five exposures of $30 \mathrm{~s}$ are chosen for the sample instead of one long exposure of $150 \mathrm{~s}$. 


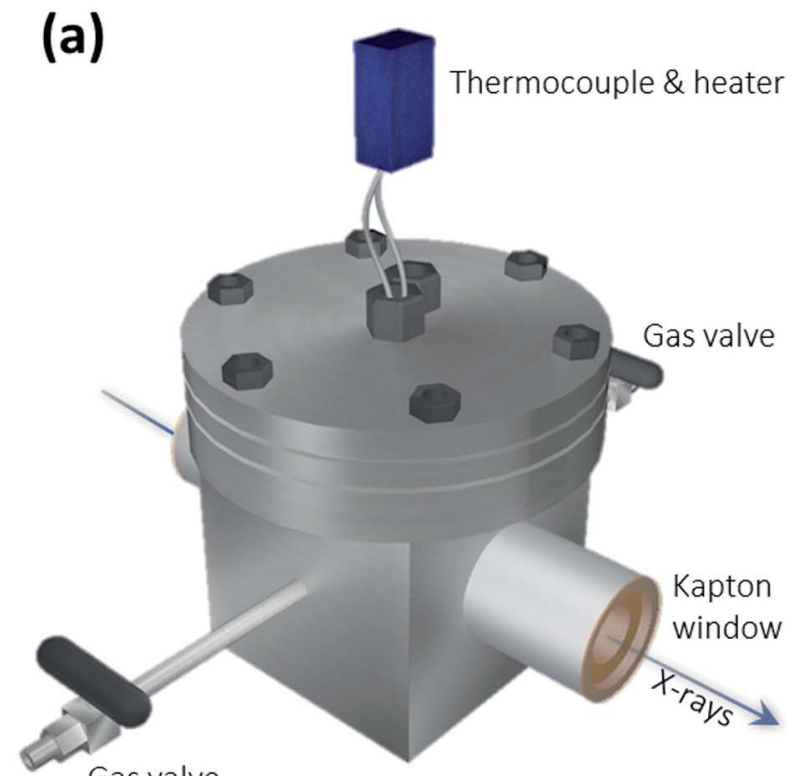

Gas valve

(b)

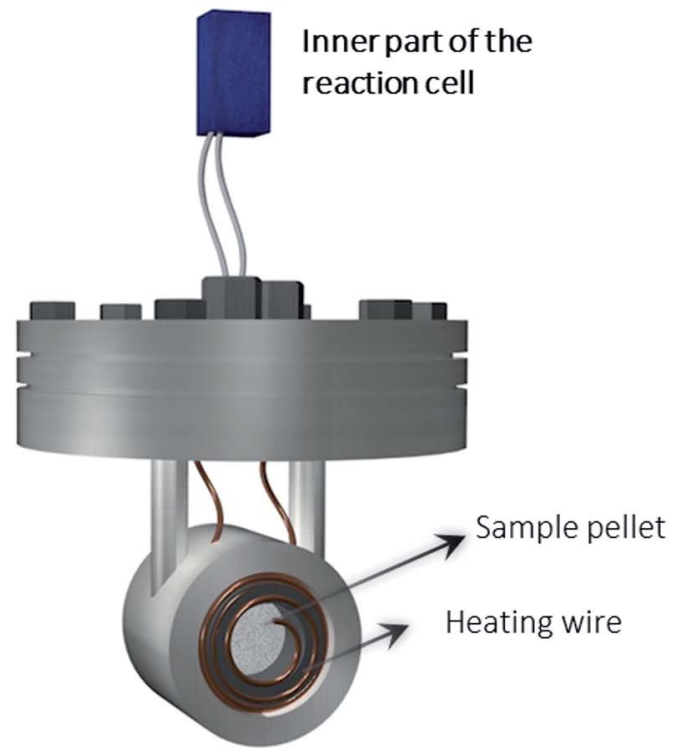

Fig. 2 3D schematics of (a) the outside and (b) the inside of the homebuilt in situ reaction cell. The reaction cell has a thermocouple, two Kapton windows, and two gas flow valves connecting to $\mathrm{H}_{2}$ or $\mathrm{N}_{2}$. The sample pellet is placed in the cylindrical holder surrounded by heating wires.

The Pd foil spectra of one particular scan and the following scan should be almost identical to ensure that the sample scan measured in between is valid. A comparison of the $i^{\text {th }}$ and the $i$ $+1)^{\text {th }}$ spectra is shown in Fig. 4. For the uncalibrated spectra, a slight drift over $75 \mathrm{~min}$ is observed; for instance, the peak at 976 pixels is slightly shifted. Nevertheless, the two consecutive scans are almost identical, meaning that there is no energy drift for the sample spectra measured in between. The details of the energy calibration will be explained in a later section. After energy calibration for each Pd foil (see Fig. 4b), all Pd spectra are almost identical.

\section{Image processing}

All images are dark-current corrected and processed with a median filter with a kernel size of $5 \times 5$ pixels to remove local outliers. Absorption images are generated on the basis of Lambert-Beer's law. Fig. 5a shows the absorption image of the Pd reference foil, calculated pixel-wise by the images $I_{\mathrm{R}}$ and $I_{0}$ shown in Fig. 3 . The unfocused XAS geometry has the advantage that the $2 \mathrm{D}$ spatial information of the sample is also imaged. Thus, additional image processing can be used to improve the final spectral quality.

To smooth the absorption image to some extent, a Gaussian low-pass filter is applied in addition to the median filter. The $\sigma$ value of the Gaussian filter is chosen as big as possible until the final energy resolution approaches the corehole lifetime broadening of Pd K-shell. The selected image filters reduce the noise level and improve the statistics. This improves the quality of the spectra without increasing the acquisition time.

Sometimes, the energies of the dispersed X-rays are not perfectly aligned, and thus the features in the absorption appear as slightly curved lines, as shown in Fig. $5 \mathrm{a}$. This is because the bending of the crystal wafer is not homogeneous. To generate the final spectrum, the absorption image is rotated and the values are averaged in the vertical direction. The deformed features may then give rise to a distorted spectrum. An image warping transformation can be performed to correct the deformation, involving the selection of some points along the curved lines (see blue points in Fig. 5a). The coordinate of a certain point along the curved line is $(x, y)$. For each point, there is a modified point lying on a corresponding straight line with a coordinate of $\left(x^{\prime}, y^{\prime}\right)$, as shown in Fig. 5b. The coordinate transformation from $(x, y)$ to $\left(x^{\prime}, y^{\prime}\right)$ can be fitted by a second-order polynomial, which, in comparison to other orders, gives the best results:

$$
\begin{aligned}
& x^{\prime}=a_{20} x^{2}+a_{02} y^{2}+a_{11} x y+a_{10} x+a_{01} y+a_{00} \\
& y^{\prime}=y
\end{aligned}
$$

The fitting coefficients $a_{\mathrm{ij}}$ are used to warp all the coordinates in Fig. 5a, resulting in a corrected absorption image as shown in Fig. $5 \mathrm{~b}$.

The 2D spatial information can also be used for a sample inhomogeneity correction, and was initially introduced for this XAS setup at the MuCLS. ${ }^{18}$ Fig. 6a shows the absorption image of the sample pellet. The X-ray footprint on the sample is relatively large. As different energies penetrate different parts of the sample, sample inhomogeneity causes a variation in the absorption signal in addition to the intensity oscillations originating from the X-ray absorption fine structure (see zoomed-in regions). To correct this, the crystal was rotated by an additional $0.2^{\circ}$ after all in situ scans. The crystal rotation shifts the energy range by $\sim 530 \mathrm{eV}$. X-ray absorption in the post-edge region is approximated by a linear function, which gives an indication of the sample's local thickness (see Fig. 6b). A thickness-corrected image can then be obtained by normalizing Fig. $6 a$ with $6 \mathrm{~b}$. The correct attenuation should be multiplied with the slope of a linear function. The inhomogeneity indicated in the zoom-in of Fig. 6a is eradicated, demonstrating the effectiveness of the method. Finally, the image is warped using the same coefficients as for Fig. 5 . The image is then 
One in-situ XAS scan (total exposure time $3.5 \mathrm{~min}$ )
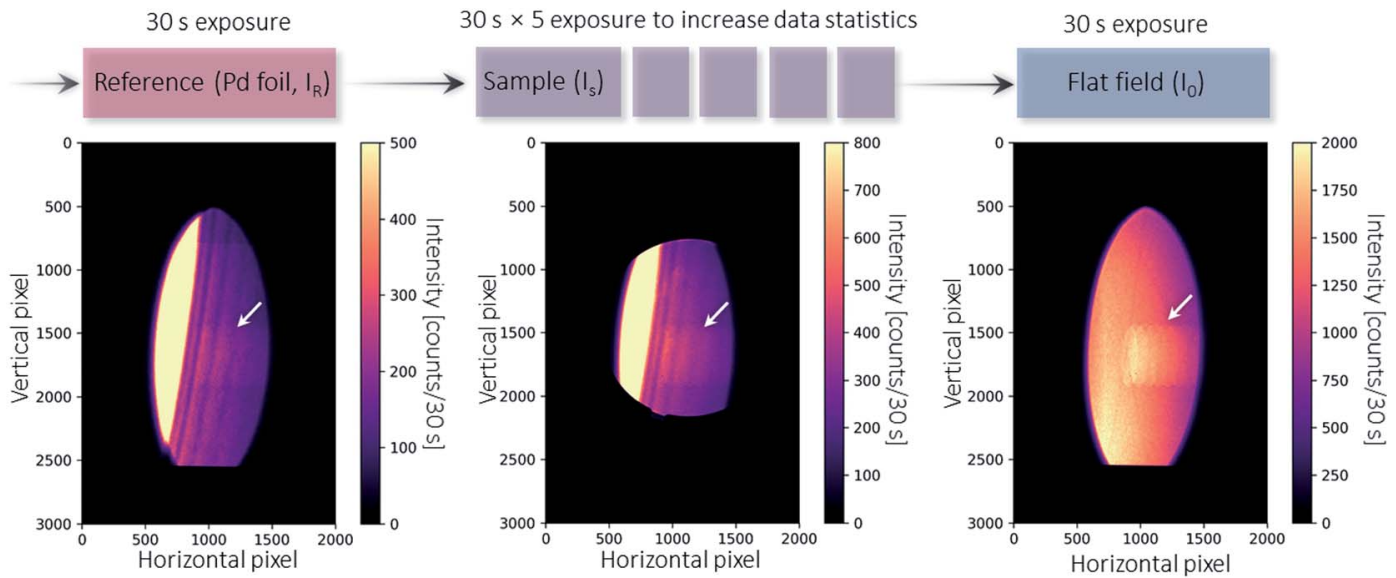

Fig. 3 Illustration of a single XAS scan during in situ temperature ramping. At the beginning of each scan, a standard reference (Pd foil, denoted as $I_{R}$ ) is taken for energy calibration and correction of temporal uncertainties between different scans (see Fig. 4). The sample is then moved in, and five exposures are acquired and averaged to increase the data statistics (denoted as $/ \mathrm{s}$ ). At the end of the scan, the sample is removed to enable a flat field image to be taken. The flat field image is used as $I_{0}$ to calculate absorption for both the reference foil and the sample. The bottom part of the beam is intercepted by the X-ray beam monitor mentioned above. ${ }^{30}$ The diffracted X-rays pass through the 3D printed crystal holder. The artifact indicated by the arrow arises from a screw thread hole in the crystal holder but is corrected when calculating the absorption using $I_{0}$ and Is.

rotated, and a polygonal region-of-interest (ROI) is marked as large as possible (see Fig. 6d), making the best use of available pixels that contain spectral information.

\section{XAS spectra generation}

Fig. 7 illustrates the energy calibration procedure. An XAS spectrum is generated by averaging all values in the ROI vertically. Several feature points were extracted for both the synchrotron reference spectrum and the measured reference spectrum. The pixel positions can be translated into X-ray energies by a second-order fitting (see eqn (2)). Fig. 7c shows the energy-calibrated spectra for the reference Pd foil. The same fitting coefficients are applied to the sample measured after the Pd foil during the same scan.
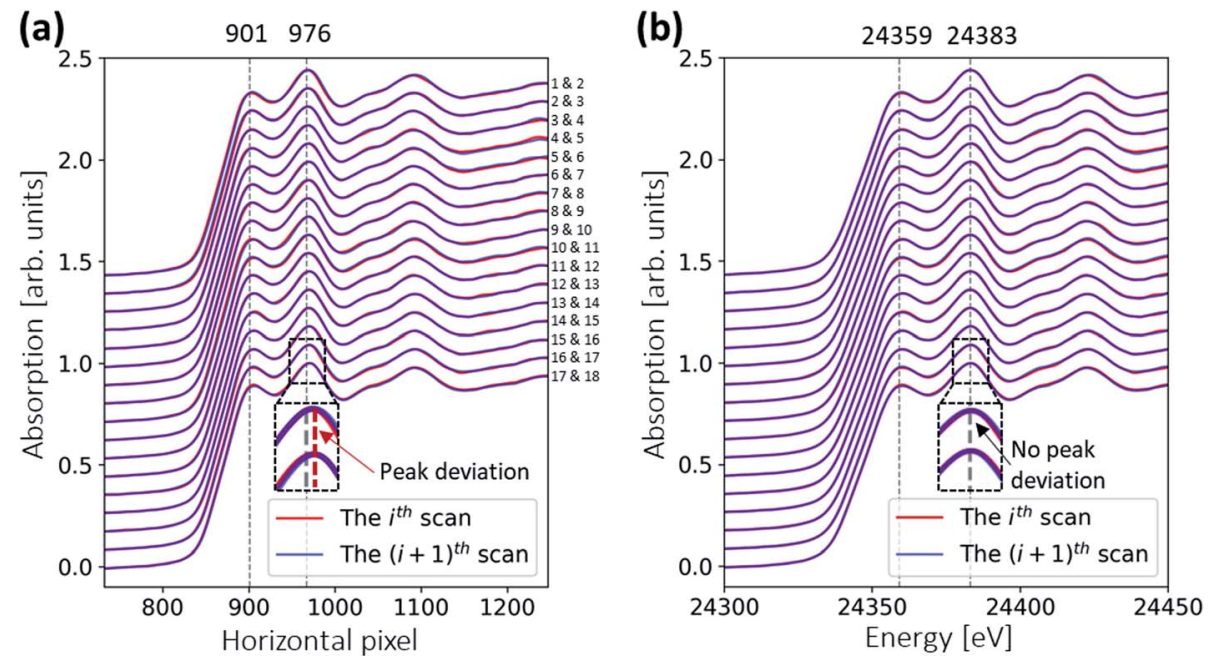

Fig. 4 Comparison of the $i^{\text {th }}$ (plotted as red curves) and $(i+1)^{\text {th }}$ (plotted as blue curves) spectra of the Pd reference foil. (a) Uncalibrated spectra. There are no observable shifts between each pair of red curves and the blue curves, meaning that there is no systematic change in a 4.3 min XAS scan, and thus the sample spectra measured in-between are accurate. However, the zoom-in of the last two spectra (the $17^{\text {th }}$ and $18^{\text {th }}$ spectra) shows that their peak position (red dotted vertical line) deviates from the peak position of the first two spectra (grey dotted line) - the energy drifts might be due to, e.g., movement of X-ray positions over 75 min. (b) Energy-calibrated spectra. Since each Pd reference spectrum is calibrated independently to the synchrotron Pd reference, the energy deviation is removed. The same calibration parameters are also used for the sample spectra measured in the same scan. 

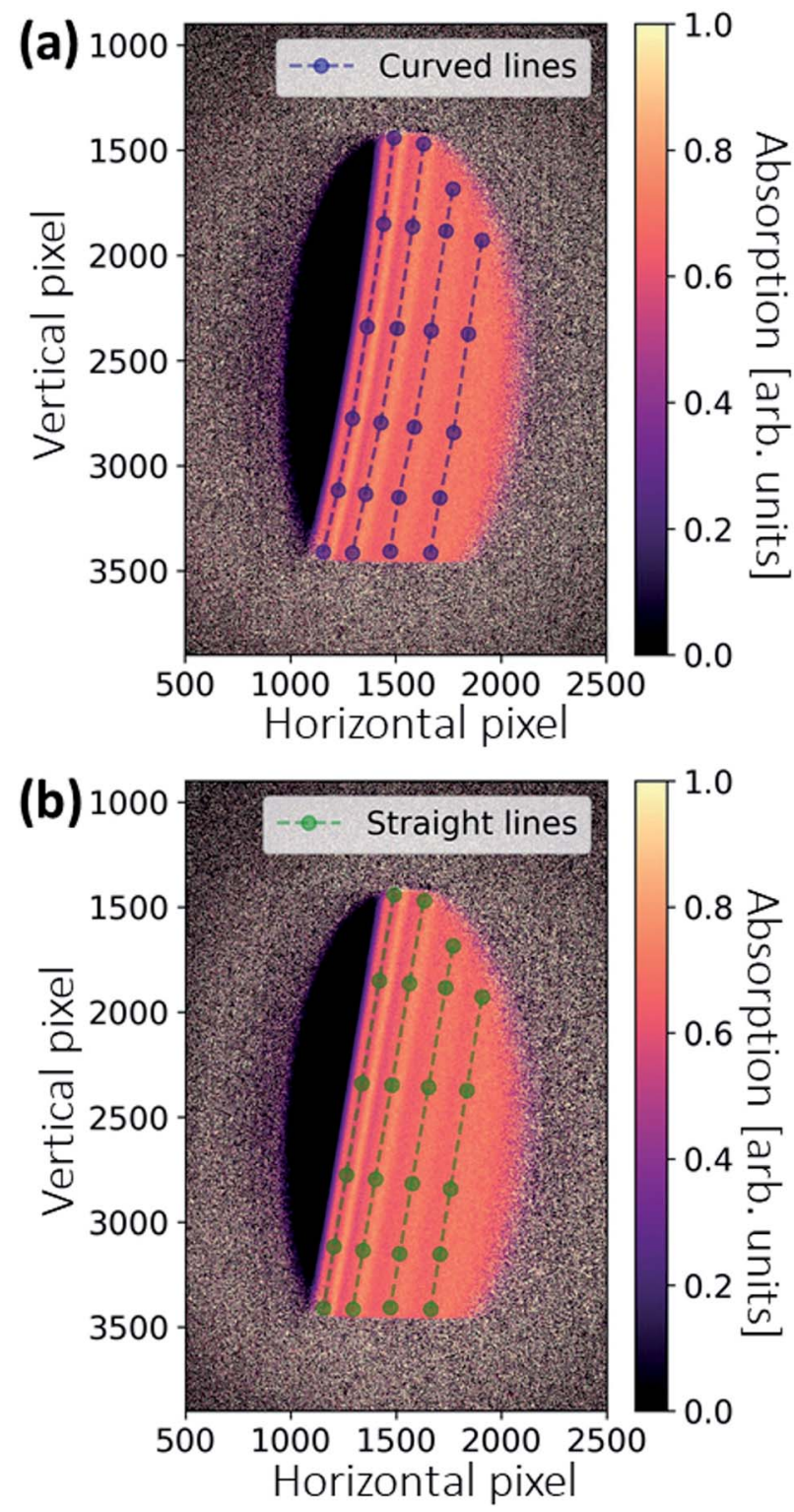

Fig. 5 Illustration of warping correction. (a) Original distorted absorption image. As a result of inhomogeneous crystal bending, the peak features in the absorption image are not on straight lines. Several points along the curved lines are selected for the warping correction. (b) Warping-corrected absorption image. In the correction, the curved feature points (blue) are fitted to the straight feature points (green) by a second-order polynomial.

$$
E=a_{2} x^{2}+a_{1} x+a_{0}
$$

where $E$ is calibrated energy values, $x$ is the horizontal pixel coordinate, and $a_{i}$ are the fitting coefficients.

\section{Energy resolution}

The energy resolution of the system is determined with raytracing calculations using the XRT package. ${ }^{44}$ Two scenarios with the only difference in the source spectra are calculated: one with a uniformly distributed spectrum and the other with a monochromatic line spectrum. The simulated results are shown in Fig. 8.

The energy resolution of the spectrometer is defined as: ${ }^{44}$

$$
\delta E=\frac{\Delta E_{\text {uni }}}{\Delta z_{\text {uni }}} \Delta z_{\text {mono }}
$$

where $\Delta E_{\text {uni }}$ is the energy range (FWHM value) covered by the diffracted beam calculated using the uniformly distributed source spectrum, $\Delta z_{\mathrm{uni}}$ and $\Delta z_{\text {mono }}$ are the diffracted beam size (FWHM values) using the uniformly distributed and monochromatic line spectrum, respectively. Parameters and Python script of the simulations can be found in the ESI. $\uparrow$ The spectrometer energy resolution is estimated to be $6.0 \mathrm{eV}$, which is smaller than the Pd K-edge lifetime broadening $6.3 \mathrm{eV}^{\mathbf{4 5}}$ Therefore, all Pd XAS features are resolved at this energy resolution.

\section{Results and discussion}

\section{Samples}

In this study, we investigated a hydrogen-pretreated $\mathrm{Pd} / \mathrm{C}$ catalyst in a phenylacetic acid decarboxylation reaction. It was observed that hydrogen pretreatment prior to reaction can significantly enhance the catalytic performance of $\mathrm{Pd} / \mathrm{C}$ even though the subsequent reaction takes place in an inert gas $\left(\mathrm{N}_{2}\right){ }^{46}$ It is known that metallic $\operatorname{Pd}(0)$ transforms into palladium hydride $\left(\mathrm{PdH}_{x}\right)$ in the presence of $\mathrm{H}_{2} \cdot{ }^{43}$ Thus, it is crucial to identify whether the formed $\mathrm{PdH}_{x}$ species during the hydrogen pretreatment can be well maintained under the decarboxylation reaction conditions. For this reason, we established this in situ XANES experiment, in which we heated up the hydrogenpretreated $\mathrm{Pd} / \mathrm{C}$ catalyst in the $\mathrm{N}_{2}$ atmosphere to investigate its chemical species under catalytic reaction conditions.

The Pd foil (thickness $25 \mu \mathrm{m}$ ), Pd/C catalyst (30 wt\%) and phenylacetic acid (purity $\geq 99.0 \%$ ) were purchased from SigmaAldrich. The phenylacetic acid was used as the reactant. The Pd/ C catalyst consists of palladium nanoparticles (NPs) supported on the activated carbon. $\mathrm{Pd} / \mathrm{C}$ sample pellets were prepared by compressing $\mathrm{Pd} / \mathrm{C}$ power with boron nitride (BN) powder (Honeywell Riedel-de Haen) at a ratio of $2: 3$ to reach optimal Xray absorption. All $\mathrm{Pd} / \mathrm{C}$ sample pellets were first activated for $30 \mathrm{~min}$ at $150{ }^{\circ} \mathrm{C}$ in $\mathrm{H}_{2}$ flow and then for another $30 \mathrm{~min}$ in $\mathrm{N}_{2}$ flow to reduce the oxidized Pd NPs to a pure $\operatorname{Pd}(0)$ state (No. 1 in Table 1). Table 1 presents the characteristics and treatments of the different samples. No. 0 refers to the reference Pd foil used to calibrate the energy and correct any temporal uncertainties between different XAS scans. No. 1 and No. 2 are the Pd/C samples treated under defined conditions, which serve as references of $\operatorname{Pd}(0)$ and $\mathrm{Pd}$ hydride forms of the sample. No. 3 is the test sample investigated in the in situ experiment. It is a hydrogen-pretreated $\mathrm{Pd} / \mathrm{C}$ sample to which the reactant, the phenylacetic acid, was added.

\section{In situ XANES results}

Fig. 9a shows the XANES spectra of the Pd standard foil (No. 0), the $\mathrm{Pd} / \mathrm{C}$ sample in $\mathrm{Pd}(0)$ form (No. 1), and in Pd hydride form 

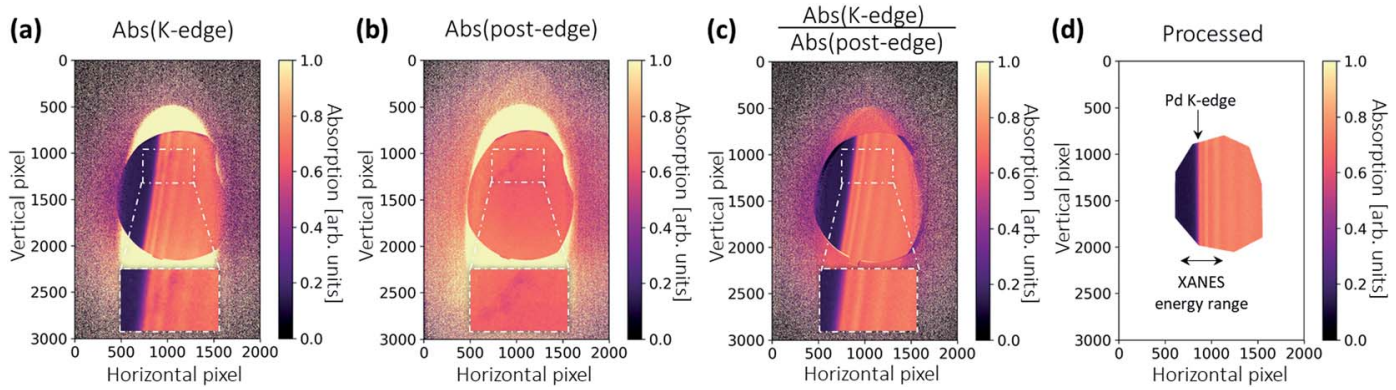

Fig. 6 Data processing for the sample. (a) An absorption image at the $\mathrm{K}$-edge, denoted as Abs(K-edge), is calculated pixel-wise from $/ 0$ and $/ \mathrm{s}$. Since the X-ray beam has a big footprint and penetrates different parts of the sample, local thickness variations are observed (see zoomed-in region). (b) An absorption image in the post-edge region, denoted as Abs(post-edge), is obtained by rotating the Laue crystal by an additional angle of $0.2^{\circ}$. (c) A thickness-corrected image is obtained by normalizing the Abs(K-edge) with the Abs(post-edge) image. (d) The absorption image in (c) is processed with the warping correction, followed by a rotation and a region-of-interest (ROI) selection.

(a)

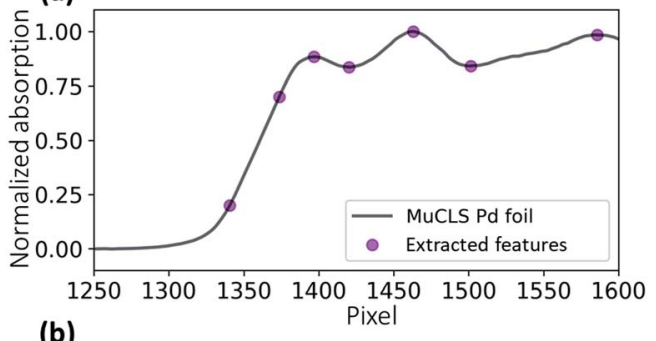

(b)

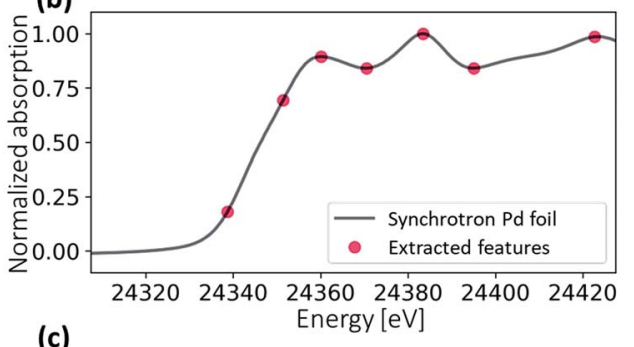

(c)

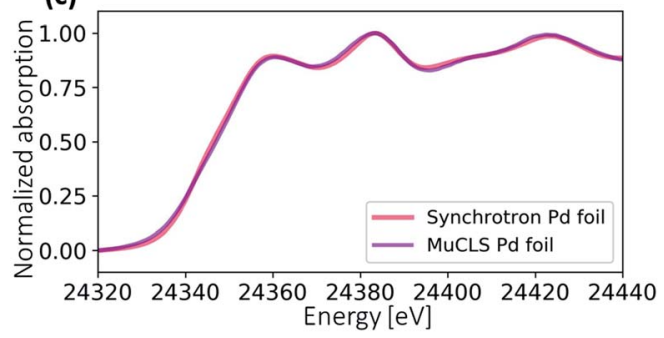

Fig. 7 Energy calibration procedure. (a) The uncalibrated spectrum of a Pd foil measured at the MuCLS. Several feature points (local extrema and edge jump points) are extracted. (b) The synchrotron spectrum of a Pd foil with extracted feature points. (c) The energy-calibrated spectrum of the Pd foil measurement at MUCLS in comparison with the synchrotron spectrum.

(No. 2), respectively. The features of sample No. 1 agree with those of the Pd foil. In contrast, spectral features of sample No. 2 are shifted towards lower energies, which is ascribed to the mixing of unoccupied states of hydrogen and palladium. ${ }^{20}$ To better visualize the difference, a difference spectrum (see the red curve around 0 in Fig. 9a) is presented, by subtracting the normalized spectrum of sample No. 1 from that of sample No.
2. The shape of this difference spectrum is consistent with the ones measured at the synchrotron. ${ }^{28}$ These three spectra serve as references for the identification of the palladium species in the decarboxylation catalytic reaction.

Fig. 9b shows the temperature ramping over $75 \mathrm{~min}$. The temperature ramping rate was set to $0.5{ }^{\circ} \mathrm{C} \mathrm{min}^{-1}$ from room temperature to $40{ }^{\circ} \mathrm{C}$ and $0.2{ }^{\circ} \mathrm{C} \mathrm{min}^{-1}$ afterwards. The reaction on the $\mathrm{Pd} / \mathrm{C}$ catalyst surface began when the temperature ramped up to certain temperature. A series of XAS spectra were collected
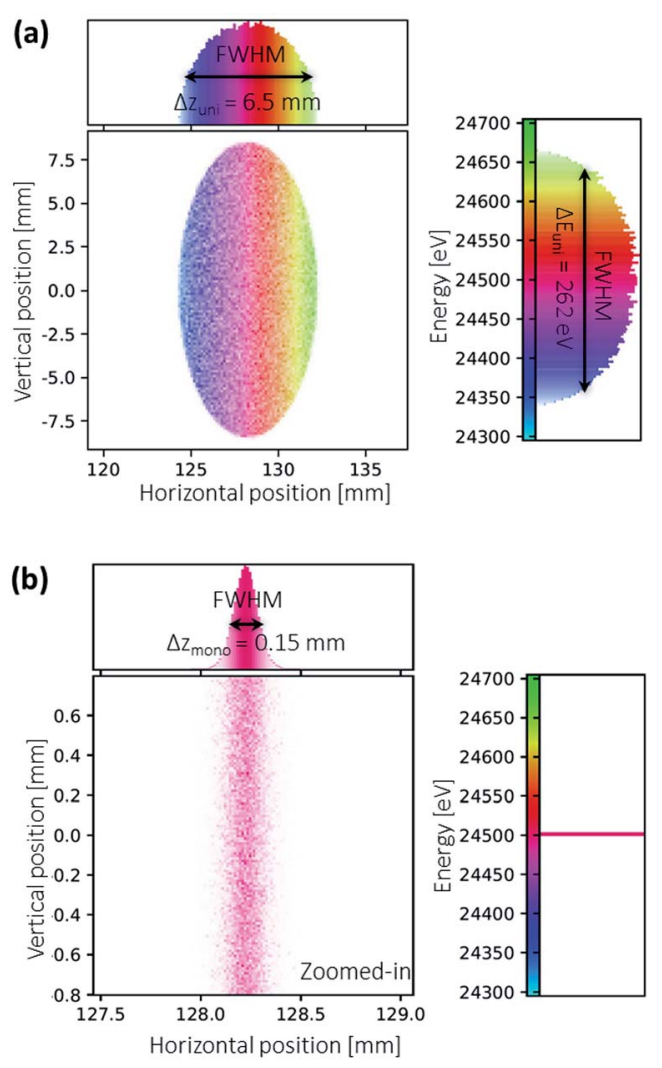

Fig. 8 Ray-traced images at the detector calculated with (a) uniformly distributed source spectrum (b) monochromatic line spectrum. The simulation geometry and Python script can be found in the ESI. $\uparrow$ 
Table 1 Details of the treatment conditions for samples before the XANES measurements

\begin{tabular}{|c|c|c|c|}
\hline No. & Sample & Used as & Treatment conditions \\
\hline 0 & Pd foil & Standard reference & $\begin{array}{l}\text { No treatment (measured in air at } \\
\text { room temperature) }\end{array}$ \\
\hline 3 & $\mathrm{Pd} / \mathrm{C}$ catalyst + phenylacetic acid & Test sample for the in situ reaction & $\begin{array}{l}\text { Based on the No. } 2 \text { treatment, the } \\
\text { Pd/C was added with the reactant. } \\
\text { (The phenylacetic acid was first } \\
\text { dissolved in hexane solvent, and } \\
\text { several droplets of the solution were } \\
\text { applied to the Pd/C pellet. The } \\
\text { hexane solvent was then vaporized, } \\
\text { leaving phenylacetic acid on the Pd/ } \\
\text { C surface. The addition of } \\
\text { phenylacetic acid was conducted in } \\
\text { the glove box.) }\end{array}$ \\
\hline
\end{tabular}

continuously using the fast scan. The start of each fast XAS scan is indicated by a differently colored dot in the temperature profile. The total acquisition time for each scan is $4.3 \mathrm{~min}$. The longer interval between the scans starting at $43 \mathrm{~min}$ and $50 \mathrm{~min}$ was due to a brief technical issue during data acquisition. At temperatures above $50{ }^{\circ} \mathrm{C}$, spectra remained virtually unchanged. Thus, only 18 scans are presented here.

Fig. 9c shows the measured XANES spectra during the temperature ramping process. The temperature at which the spectra were acquired is color-coded in Fig. 9c according to the colors used in Fig. 9b. Pd/C reference samples in Pd hydride (sample No. 2) and $\mathrm{Pd}(0)$ form (sample No. 1) are plotted above and below the in situ spectra for comparison. The difference spectra of the sample and the Pd foil - the difference between each in situ scan and the averaged spectrum of the last three spectra of the entire series - are also plotted in Fig. 9c. The difference spectra of the Pd reference foil have small and random oscillations, demonstrating that the spectral change observed in samples is indeed due to chemical species change rather than systematic fluctuations from the instruments.

At the beginning of the in situ experiment, the spectrum undergoes a shift towards lower energy that is similar to the
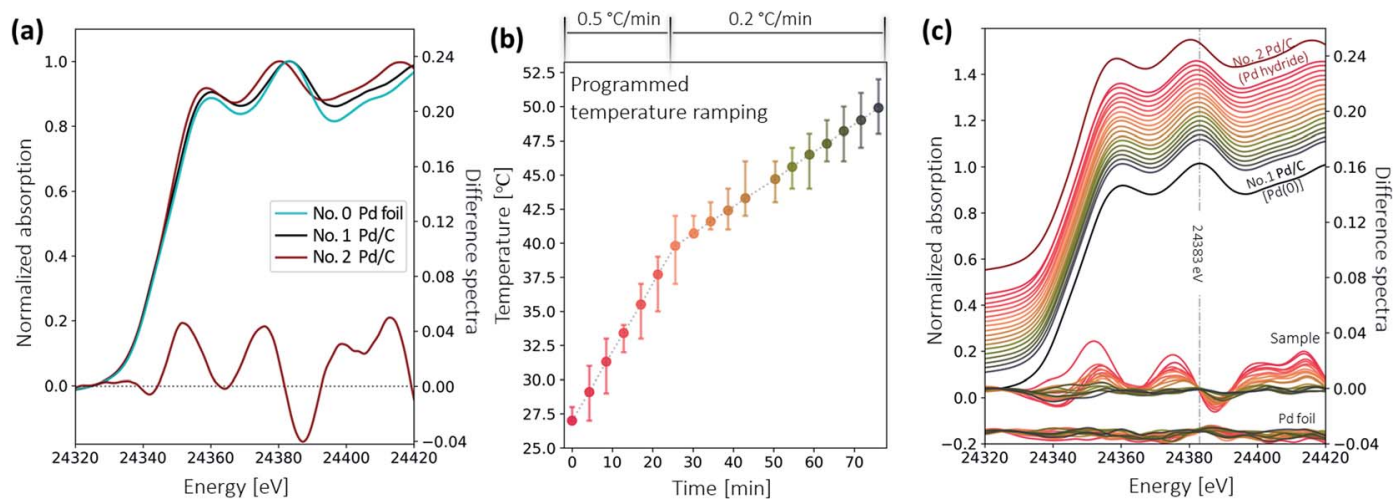

Fig. 9 (a) XANES spectra of reference No. 0,1, 2 (Pd foil, $\mathrm{Pd} / \mathrm{C}$ catalyst in the form of bare $\mathrm{Pd}(0)$ ), $\mathrm{Pd} / \mathrm{C}$ catalyst in the form of $\mathrm{PdH} \mathrm{H}_{x}$ ). The red curve around zero presents the difference between the No. 1 and the No.2 sample. This highlights the subtle differences between the two XANES spectra. (b) The temperature ramping profile, ramping from room temperature to $50^{\circ} \mathrm{C}$ over $75 \mathrm{~min}$. Set temperature values are plotted as dots, while the measured temperature ranges during the individual acquisitions are plotted as error bars. (c) Corresponding Pd XANES spectra measured at different temperatures. The normalized spectra were shifted vertically with an arbitrary offset from one another for readability. The spectra of the No. 1 and No. 2 Pd/C catalyst are plotted below (black) and above (red) the in situ spectra for comparison. The difference between each XANES spectrum and the last three averaged XANES spectra of the entire series are displayed around 0 and -0.04 for the sample and the Pd foil, respectively. No significant changes are observed in the Pd foil difference spectra, which confirms that the spectral changes of the sample are due to its chemical changes. A Savitzky-Golay moving average filter was applied to the difference spectra. 
behavior of the Pd hydride reference (sample No. 2). But the shift is smaller than that in the Pd hydride reference, which is also confirmed by the smaller amplitude of the difference spectrum. This indicates that the $\mathrm{Pd} / \mathrm{C}$ sample was initially still in the Pd hydride form after adding the reactant, but with fewer hydrogen atoms incorporated into the Pd lattice. While ramping up the temperature, a slight shift towards higher energy is observed. The difference spectra around 0 present more clearly the subtle changes that are hard to see directly from the spectra. The shape of the spectra gradually evolves into a shape resembling the bare $\mathrm{Pd}(0)$ form of the $\mathrm{Pd} / \mathrm{C}$ (sample No. 1) and becomes nearly identical at $50{ }^{\circ} \mathrm{C}$. No changes are observed as the reaction temperature increases further.

The in situ XAS results reveal that the hydrogen pretreatment can initially transfer the $\mathrm{Pd}(0)$ species to the $\mathrm{PdH}_{x}$ species, and this $\mathrm{PdH}_{x}$ can be maintained with the addition of reactant at room temperature. However, $\mathrm{PdH}_{x}$ species surfers a decomposition process when ramping up the temperature.

To further exclude the possibility that the spectral change was caused by consumption of the limited sample amount, online mass spectrometry and static XANES were further conducted. A temperature-programmed surface reaction (TPSR) on No. 3 sample with online mass-spectrometry was conducted with the same temperature ramping speed. The TPSR result shows that the reaction rate significantly speeds up at a temperature of $90{ }^{\circ} \mathrm{C}$, well above the $50{ }^{\circ} \mathrm{C},{ }^{46}$ meaning that the transform of chemical species is completed before the catalytic reaction becomes most active. The additional static XAS experiment was carried out on a different day, in which the temperature was heated up to $100{ }^{\circ} \mathrm{C}$ within $10 \mathrm{~min}$, and a XANES spectrum was measured immediately. The same trend of spectra shapes changes from $\mathrm{PdH}_{x}$ to $\mathrm{Pd}(0)$ was observed. Both the mass spectrometry and static XANES experiments confirm that the spectral changes are due to the chemical species change of the $\mathrm{Pd} / \mathrm{C}$ catalyst.

Since transmission-mode XAS is a bulk-sensitive technique that provides averaged information of the test pellet, the spectral shape above $50{ }^{\circ} \mathrm{C}$ cannot provide direct evidence of whether the small amount of hydrogen is still incorporated in the Pd lattice. Therefore, combining the in situ XANES results and the fact that hydrogen-pretreated $\mathrm{Pd} / \mathrm{C}$ has significantly better catalytic performance than bare Pd/C (sample No. 1), ${ }^{\mathbf{4 6}}$ the hydrogen-pretreated $\mathrm{Pd} / \mathrm{C}$ is concluded to be an $\alpha-\mathrm{PdH}_{x}$ species under reaction conditions, as its spectral shapes highly resemble that of bare $\operatorname{Pd}(0) .{ }^{27}$ And the enhanced catalytic activity of the hydrogen-pretreated $\mathrm{Pd} / \mathrm{C}$ is highly correlated with a formation of $\alpha$-phase $\mathrm{PdH}_{x}$ rather than bare $\operatorname{Pd}(0)$.

\section{Conclusions}

In this study, we performed an in situ XAS experiment on a $\mathrm{Pd} / \mathrm{C}$ catalyst at the MuCLS facility. We investigated the active species of the hydrogen-pretreated $\mathrm{Pd} / \mathrm{C}$ catalyst in the carboxylic acid decarboxylation reaction. To this end, we measured a series of Pd K-edge XANES of the sample, to which phenylacetic acid was added when ramping up the temperature in the $\mathrm{N}_{2}$ atmosphere. We observed a dynamic spectral change of Pd hydride species to a spectral shape resembling $\operatorname{Pd}(0)$ form between room temperature and $50{ }^{\circ} \mathrm{C}$. Combining TPSR and in situ XANES results, we believe that the active species of the hydrogen pretreated $\mathrm{Pd} / \mathrm{C}$ catalyst for the decarboxylation reaction should be $\alpha$-phase $\mathrm{PdH}_{x}$ - only a small amount of hydrogen is incorporated in the Pd lattice-rather than metallic $\operatorname{Pd}(0)$.

The synchrotron-comparable XANES collected at the compact ICS facility is sufficiently sensitive to distinguish the subtle differences between Pd and Pd hydride at a time resolution of less than $5 \mathrm{~min}$ and an energy resolution below the Pd K-hole lifetime broadening. Several advantages over other compact systems exist at MuCLS for in situ XAS experiments in terms of data collection efficiency, spectral quality, source stability, flexibility of the operating space, and high X-ray energy. First, the higher spectral flux, smaller bandwidth and smaller divergence contribute to an efficient XAS data acquisition, allowing for in situ measurements on a time scale of only minutes in the high-energy X-ray regime $>\mathbf{1 5} \mathrm{keV}$, or even less when a degraded spectral quality is acceptable. Second, the well-defined source spectrum and the energy tunability allow synchrotron-comparable XAS spectra to be generated with a high signal-to-noise ratio. Third, the stability of the X-ray source at the MuCLS satisfies the requirement of long-term experimentation. Fourth, the separate experiment hutch provides sufficient operating space for the implementation of complex sample environments. Finally, high-energy X-rays facilitate in situ/in operando setups in various atmospheres due to reduced $\mathrm{X}$-ray absorption by the atmospheres and sample environments. However, there are also some drawbacks. First, the X-ray energy at the MuCLS is currently only tunable from $15 \mathrm{keV}$ to $35 \mathrm{keV}$, which restricts the elements that can be investigated to this energy range - though pushing the energy range to a lower limit is theoretically possible. Second, the dispersive setup restricts measurement to transmission mode only. Therefore, dilute samples are challenging to measure.

To summarize, these first in situ XAS results obtained at an ICS facility demonstrate the great potential of such sources for in situ XAS experiments, which are usually conducted at large facilities. With an intermediate cost and performance between those of an X-ray tube and a synchrotron, such laboratory-scale facilities can provide excellent alternatives for in situ XAS techniques and advance their widespread use in science and industry.

\section{Author contributions}

FP, MD, AJ, YL and JH conceived the experiments. JH, FD, MD, YL and AJ performed the experiments. FP, MD, BG and KA contributed to the experiments including the ICS source installation at the Munich Compact Light Source. JH, FD and YL analyzed the data. JH and FD wrote the manuscript with significant input from all co-authors.

\section{Conflicts of interest}

There are no conflicts to declare. 


\section{Acknowledgements}

We wish to acknowledge the financial support by the Center for Advanced Laser Applications (CALA). This study was supported by the German Research Foundation (DFG) and the Technical University of Munich (TUM) within the framework of the Open Access Publishing Program. We would also like to thank the staff of Lyncean Technologies Inc. for their technical support. Further, we wish to acknowledge Dr Yitao Cui and the SPring-8 BL14B2 XAFS database for the Pd metal standard spectrum. FD would like to thank the Chinese Scholarship Council (CSC) for the financial support. JH wishes to thank Suzana Spasova for her assistance during the easurements.

\section{References}

1 D. C. Koningsberger and R. Prins, X-ray Absorption: Principles, Applications, Techniques of EXAFS and XANES, Wiley, New York, 1988, vol.92.

2 J. Yano and V. K. Yachandra, Photosynth. Res., 2009, 102, 241254.

3 F. W. Lytle, J. Synchrotron Radiat., 1999, 6, 123-134.

4 R. Bès, T. Ahopelto, A. P. Honkanen, S. Huotari, G. Leinders, J. Pakarinen and K. Kvashnina, J. Nucl. Mater., 2018, 507, 5053.

5 A. S. Ditter, E. P. Jahrman, L. R. Bradshaw, X. Xia, P. J. Pauzauskie and G. T. Seidler, J. Synchrotron Radiat., 2019, 26, 2086-2093.

6 Z. Németh, J. Szlachetko, É. G. Bajnóczi and V. György, Rev. Sci. Instrum., 2016, 87, 103105.

7 W. Błachucki, J. Czapla-Masztafiak, J. Sá and J. Szlachetko, J. Anal. At. Spectrom., 2019, 34, 1409-1415.

8 F. Zeeshan, J. Hoszowska, L. Loperetti-Tornay and J.-C. Dousse, Rev. Sci. Instrum., 2019, 90, 73105.

9 C. Schlesiger, S. Praetz, R. Gnewkow, W. Malzer and B. Kanngießer, J. Anal. At. Spectrom., 2020, 35, 2298-2304.

10 P. Zimmermann, S. Peredkov, P. M. Abdala, S. DeBeer, M. Tromp, C. Müller and J. A. van Bokhoven, Coord. Chem. Rev., 2020, 423, 213466.

11 W. Malzer, C. Schlesiger and B. Kanngießer, Spectrochim. Acta, Part B, 2021, 177, 106101.

12 J. G. Moya-Cancino, A.-P. Honkanen, A. M. J. van der Eerden, H. Schaink, L. Folkertsma, M. Ghiasi, A. Longo, F. M. F. de Groot, F. Meirer, S. Huotari and B. M. Weckhuysen, ChemCatChem, 2019, 11, 1039-1044.

13 K. Dupraz, et al., Phys. Open, 2020, 5, 100051.

14 P. Cardarelli, A. Bacci, R. Calandrino, F. Canella, R. Castriconi, S. Cialdi, A. Del Vecchio, F. Di Franco, I. Drebot, M. Gambaccini, D. Giannotti, A. Loria, G. Mettivier, G. Paternò, V. Petrillo, M. Rossetti Conti, P. Russo, A. Sarno, E. Suerra, A. Taibi and L. Serafini, Phys. Med., 2020, 77, 127-137.

15 Z. Chi, L. Yan, Y. Du, Z. Zhang, W. Huang, H. Chen and C. Tang, Nucl. Instrum. Methods Phys. Res., Sect. B, 2017, 402, 364-369.

16 W. Graves, J. Chen, P. Fromme, M. Holl, K.-H. Hong, R. Kirian, C. Limborg-Deprey, L. Malin, D. Moncton, E.
Nanni, K. Schmidt, J. Spence, M. Underhill, U. Weierstall, N. Zatsepin and C. Zhang, ASU Compact XFEL, JACoW, Geneva, Switzerland, 2018.

17 C. Vaccarezza, et al., Nucl. Instrum. Methods Phys. Res., Sect. A, 2016, 829, 237-242.

18 J. Huang, B. Günther, K. Achterhold, Y.-T. Cui, B. Gleich, M. Dierolf and F. Pfeiffer, Sci. Rep., 2020, 10, 8772.

19 F. Meier, L. Zhou, J. Wiebe and R. Wiesendanger, Science, 2008, 320, 82-86.

20 A. L. Bugaev, A. A. Guda, I. A. Pankin, E. Groppo, R. Pellegrini, A. Longo, A. V. Soldatov and C. Lamberti, Catal. Today, 2019, 336, 40-44.

21 S. Zhao, Y. Li, D. Liu, J. Liu, Y.-M. Liu, D. N. Zakharov, Q. Wu, A. Orlov, A. A. Gewirth, E. A. Stach, R. G. Nuzzo and A. I. Frenkel, J. Phys. Chem. C, 2017, 121, 18962-18972.

22 C. He, J. Tao, Y. Ke and Y. Qiu, RSC Adv., 2015, 5, 6669566703.

23 C. Zhao, T. Brück and J. A. Lercher, Green Chem., 2013, 15, 1720.

24 S. Lestari, P. Mäki-Arvela, H. Bernas, O. Simakova, R. Sjöholm, J. Beltramini, G. Q. M. Lu, J. Myllyoja, I. Simakova and D. Y. Murzin, Energy Fuels, 2009, 23, 3842-3845.

25 S. Lestari, I. Simakova, A. Tokarev, P. Mäki-Arvela, K. Eränen and D. Y. Murzin, Catal. Lett., 2008, 122, 247-251.

26 M. W. Tew, M. Janousch, T. Huthwelker and J. A. van Bokhoven, J. Catal., 2011, 283, 45-54.

27 A. L. Bugaev, A. A. Guda, K. A. Lomachenko, V. V. Srabionyan, L. A. Bugaev, A. V. Soldatov, C. Lamberti, V. P. Dmitriev and J. A. van Bokhoven, J. Phys. Chem. C, 2014, 118, 10416-10423.

28 A. L. Bugaev, A. A. Guda, A. Lazzarini, K. A. Lomachenko, E. Groppo, R. Pellegrini, A. Piovano, H. Emerich, A. V. Soldatov, L. A. Bugaev, V. P. Dmitriev, J. A. van Bokhoven and C. Lamberti, Catal. Today, 2017, 283, 119-126. 29 A. L. Bugaev, O. A. Usoltsev, A. Lazzarini, K. A. Lomachenko, A. A. Guda, R. Pellegrini, M. Carosso, J. G. Vitillo, E. Groppo, J. A. van Bokhoven, A. V. Soldatov and C. Lamberti, Faraday Discuss., 2018, 208, 187-205.

30 B. Günther, M. Dierolf, K. Achterhold and F. Pfeiffer, J. Synchrotron Radiat., 2019, 26, 1546-1553.

31 B. Günther, R. Gradl, C. Jud, E. Eggl, J. Huang, S. Kulpe, K. Achterhold, B. Gleich, M. Dierolf and F. Pfeiffer, J. Synchrotron Radiat., 2020, 27, 1395-1414.

32 E. Eggl, M. Dierolf, K. Achterhold, C. Jud, B. Günther, E. Braig, B. Gleich and F. Pfeiffer, J. Synchrotron Radiat., 2016, 23, 1137-1142.

33 P. Glatzel, T.-C. Weng, K. Kvashnina, J. Swarbrick, M. Sikora, E. Gallo, N. Smolentsev and R. A. Mori, J. Electron Spectrosc. Relat. Phenom., 2013, 188, 17-25.

34 P. Lecante, J. Jaud, A. Mosset, J. Galy and A. Burian, Rev. Sci. Instrum., 1994, 65, 845-849.

35 U. Kaminaga, T. Matsushita and K. Kohra, Jpn. J. Appl. Phys., 1981, 20, L355-L358.

36 S. Pascarelli, O. Mathon, M. Muñoz, T. Mairs and J. Susini, J. Synchrotron Radiat., 2006, 13, 351-358. 
37 C. Schlesiger, L. Anklamm, H. Stiel, W. Malzer and B. Kanngießer, J. Anal. At. Spectrom., 2015, 30, 1080-1085.

38 A. Kulow, S. Witte, S. Beyer, A. G. Buzanich, M. Radtke, U. Reinholz, H. Riesemeier and C. Streli, J. Anal. At. Spectrom., 2019, 34, 239-246.

39 R. Gradl, M. Dierolf, L. Hehn, B. Günther, A. Ö. Yildirim, B. Gleich, K. Achterhold, F. Pfeiffer and K. S. Morgan, Sci. Rep., 2017, 7, 4908.

40 C. Jud, E. Braig, M. Dierolf, E. Eggl, B. Günther, K. Achterhold, B. Gleich, E. Rummeny, P. Noël, F. Pfeiffer and D. Muenzel, Sci. Rep., 2017, 7, 14477.

41 E. Eggl, S. Grandl, A. Sztrókay-Gaul, M. Dierolf, C. Jud, L. Heck, K. Burger, B. Günther, K. Achterhold, D. Mayr, J. J. Wilkens, S. D. Auweter, B. Gleich, K. Hellerhoff,
M. F. Reiser, F. Pfeiffer and J. Herzen, Sci. Rep., 2018, 8, 15700.

42 S. Kulpe, M. Dierolf, B. Günther, M. Busse, K. Achterhold, B. Gleich, J. Herzen, E. Rummeny, F. Pfeiffer and D. Pfeiffer, Sci. Rep., 2019, 9, 13332.

43 A. Baldi, T. C. Narayan, A. L. Koh and J. A. Dionne, Nat. Mater., 2014, 13, 1143-1148.

44 K. Klementiev and R. Chernikov, Proc. SPIE, 2014, 92090A. 45 J. L. Campbell and T. Papp, At. Data Nucl. Data Tables, 2001, 77, 1-56.

46 F. Deng, J. Huang, E. A. Ember, K. Achterhold, M. dierolf, A. Jentys, Y. Liu, F. Pfeiffer and J. A. Lercher, 2021, Submitted. 